\title{
Pangenome analyses of the wheat pathogen Zymoseptoria tritici reveal the structural basis of a highly plastic eukaryotic genome
}

\author{
Clémence Plissonneau ${ }^{1,2}$, Fanny E. Hartmann ${ }^{1,3}$ and Daniel Croll ${ }^{4^{*}}$ (D)
}

\begin{abstract}
Background: Structural variation contributes substantially to polymorphism within species. Chromosomal rearrangements that impact genes can lead to functional variation among individuals and influence the expression of phenotypic traits. Genomes of fungal pathogens show substantial chromosomal polymorphism that can drive virulence evolution on host plants. Assessing the adaptive significance of structural variation is challenging, because most studies rely on inferences based on a single reference genome sequence.

Results: We constructed and analyzed the pangenome of Zymoseptoria tritici, a major pathogen of wheat that evolved host specialization by chromosomal rearrangements and gene deletions. We used single-molecule real-time sequencing and high-density genetic maps to assemble multiple genomes. We annotated the gene space based on transcriptomics data that covered the infection life cycle of each strain. Based on a total of five telomere-to-telomere genomes, we constructed a pangenome for the species and identified a core set of 9149 genes. However, an additional 6600 genes were exclusive to a subset of the isolates. The substantial accessory genome encoded on average fewer expressed genes but a larger fraction of the candidate effector genes that may interact with the host during infection. We expanded our analyses of the pangenome to a worldwide collection of 123 isolates of the same species. We confirmed that accessory genes were indeed more likely to show deletion polymorphisms and loss-of-function mutations compared to core genes.
\end{abstract}

Conclusions: The pangenome construction of a highly polymorphic eukaryotic pathogen showed that a single reference genome significantly underestimates the gene space of a species. The substantial accessory genome provides a cradle for adaptive evolution.

Keywords: Fungal pathogen, Genome evolution, Genome assembly, Pangenome analyses, Pathogen evolution, Zymoseptoria tritici

\section{Background}

Chromosomal rearrangements facilitate the emergence of evolutionary novelty in eukaryotic genomes [1]. Among other consequences, rearrangements impact adaptive evolution by generating variation in gene content among individuals and through the emergence of new genes. Most gene gains are the result of duplication events, followed by

\footnotetext{
* Correspondence: daniel.croll@unine.ch

${ }^{4}$ Laboratory of Evolutionary Genetics, Institute of Biology, University of Neuchâtel, CH-2000 Neuchâtel, Switzerland

Full list of author information is available at the end of the article
}

diversification and neofunctionalization [2, 3]. Nonhomologous recombination can lead to gains in gene function through the acquisition of new domains. Gene gains have been traced back to horizontal gene transfer events or to de novo gene emergence from non-coding DNA [4, 5]. Gene losses can occur through nonhomologous recombination or pseudogenization. Despite being largely under negative selection, gene losses also contribute to adaptive evolution [6]. Gene gains and losses are critical for the rapid adaptation of filamentous plant pathogens to different hosts [7]. 
In fungi, intra- and interspecific structural variation among genomes has long been recognized [8]. In pathogens, structural variation can have an impact on host range. For example, the host jump from dicotyledon to monocotyledon hosts was accompanied by the loss of genes in the fungus Melanopsichium pennsylvanicum [9]. Over longer evolutionary time scales, the expansion or contraction of gene families is tightly associated with host specialization of plant pathogenic fungi [10-12]. In contrast to the ubiquitous evidence for structural variation among genomes of different species, intraspecific analyses of structural variation and polymorphism in gene content are rare outside of a small number of model organisms. Yet, many fungal genomes are amenable to accurate and complete genome assemblies using long-read sequencing technologies [13, 14].

Analyses of multiple complete genomes of the same species have the potential to reveal segregating chromosomal polymorphism and are necessary to accurately cover the gene repertoire of a species. Capturing the full gene repertoire of a species is widely referred to as the pangenome $[15,16]$. The pangenome concept distinguishes the core genome (i.e., genes found in all individuals) from the accessory genome (i.e., genes absent in one or more individuals). The distinction between core and dispensable genomic regions is relevant because these compartments are often on distinct evolutionary trajectories [17-19]. Pangenomes constructed over the past decade revolutionized the understanding of genomic variability in bacterial species or lineages. For example, the sequencing of 61 Escherichia coli strains showed that only 993 genes are shared among all individuals, while the pangenome is estimated to comprise 15,741 genes [20]. Compared to the relative simplicity of prokaryotic genomes, advances in long read sequencing technologies have been necessary to efficiently assemble eukaryotic genomes [21, 22]. Recently, pangenomes have been constructed for plant species with complex genomes, including maize, Brassica oleracea, and soybean species [23-25]. Intra-species genomic analyses of fungi have largely focused on species complexes and on genes missing compared to a reference genome strain [26-29], with the notable exception of Baker's yeast, where 12 complete genome assemblies are available [30].

Capturing the intra-specific gene content is particularly relevant for plant pathogenic fungi because major determinants of pathogenicity are often encoded by genes not shared among all strains. Plant pathogens and their hosts are often locked in arms races to counter newly evolved host resistance and fungal pathogenicity factors, respectively [31]. To enhance plant colonization, pathogens secrete small effector molecules, which can be recognized by plant resistance proteins to trigger defense mechanisms [32]. In modern agroecosystems, the large-scale deployment of single host genotypes can create uniformity in host resistance mechanisms. Such monocultures exert strong selection pressures on pathogens to evolve effectors that target the deployed host resistance mechanism [33]. Analyses of a broad range of plant pathogens revealed that these rapidly evolving effectors were often located in rapidly evolving compartments of the genome [34]. In filamentous plant pathogens, the compartmentalization of the genome and tight association of effectors and transposable elements in the same compartments has been described as the 'two-speed genome' model of pathogen evolution [7]. This bipartite compartmentalization includes blocks of conserved and gene-dense regions and blocks of highly rearranged regions, which are generally gene-poor but enriched in rapidly evolving effector loci. Capturing the pangenome of a pathogen species will enable joint analyses of genome plasticity and yet unknown determinants of pathogenicity.

The plant pathogenic fungus Zymoseptoria tritici is responsible for Septoria tritici blotch, one of the most damaging diseases on wheat [35]. Z. tritici populations have rapidly evolved resistance to fungicides and have surmounted major wheat resistance genes [36, 37]. Rapid adaptive evolution was likely facilitated by the large effective population sizes, gene flow, and high recombination rates [38-40]. However, the genetic basis of virulence remains poorly understood and, to date, only a few effector genes have been functionally characterized [41-43]. The genome of $Z$. tritici harbors 13 core chromosomes and up to 8 accessory chromosomes, which are not found in all strains of the species. Accessory chromosomes have been shown to undergo major structural rearrangements during meiosis $[44,45]$. Homologous core chromosomes show substantial length polymorphism, which is caused by the insertion or deletion of clusters of transposable elements [14, 46].

Gene content can vary considerably among homologous chromosomes. Analyses of two completely assembled genomes showed that hundreds of genes were unique to either of the two genomes $[14,45]$. Chromosomal sequences harboring such orphan regions within species (i.e., not shared among all isolates) were also referred to as accessory or dispensable regions [14, 47]. Orphan regions play an important role in adaptive evolution of the pathogen as these regions are enriched in effector genes, including the recently discovered effectors linked to the breakdown of host resistance [42, 48].

We constructed a pangenome of $Z$. tritici by performing de novo complete genome assemblies and analyses of a total of five telomere-to-telomere genomes. Using comprehensive transcriptomics datasets, we annotated the gene space of each newly assembled genome and performed comparative genomics analyses to estimate 
the total gene content of the species. We extrapolated from the pangenome of five strains to the entire species by analyzing genome-wide polymorphism in a worldwide collection of 123 sequenced isolates.

\section{Results}

\section{Complete genome assemblies of $Z$. tritici strains}

We used high-coverage PacBio sequencing to assemble complete chromosomes of the $Z$. tritici strains $1 \mathrm{~A} 5$, 3D1, and 3D7. These three strains and the two strains for which complete genomes were already available had approximately identical phylogenetic distances (Fig. 1a). A principal component analysis revealed that strains $1 \mathrm{~A} 5,1 \mathrm{E} 4,3 \mathrm{D} 1$, and 3D7 were genetically more similar to each other than the reference genome isolate IPO323 (Fig. 1b) reflecting the geographic origin of the different strains. High-density genetic maps available for the same isolates [49] confirmed the contiguity of all assembled chromosomes. Illumina read mapping to each genome assembly revealed $\leq 12$ single base or small indel errors, which were corrected according to the evidence from the Illumina read data. The three new genomes comprised 20 (1E4) or 21 (1A5 and 3D1) chromosomes (Fig. 1c). The two previously available genomes comprised 17 (3D7) and 21 chromosomes (reference genome IPO323). The total size of the five genomes ranged from 37.9 to $40.7 \mathrm{Mb}$ (Additional file 1: Table S1). Differences in genome sizes were due to a combination of differences in accessory chromosome numbers (4-8) and chromosome length polymorphism (Fig. 1c). Core chromosome length polymorphism ranged from $2.1 \%$ to $4.8 \%$, whereas length polymorphism among accessory chromosomes reached up to 25\% (Additional file 1: Table S1). Length variation among homologous chromosomes was caused by substantial insertion and deletion polymorphism in both genic and non-genic regions (Fig. 2).

\section{Gene prediction and comparative analyses of gene content}

Comparative genomics analyses of the two previously completed genomes 3D7 and IPO323 conservatively estimated that each genome harbored 296 and 216 gene sequences, respectively, that had no homology in the other genome [14]. To identify protein coding genes in the assembled genomes, we performed gene prediction analyses using splicing evidence from transcriptomic datasets collected from each of the isolates over the a

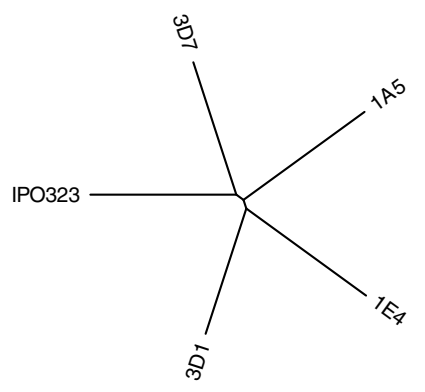

$\overline{0.05}$ b

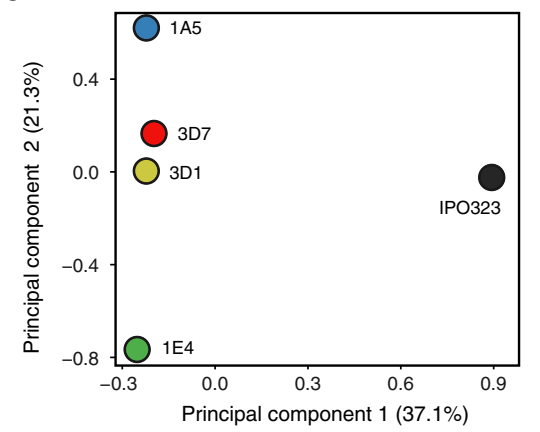

C

Core chromosomes
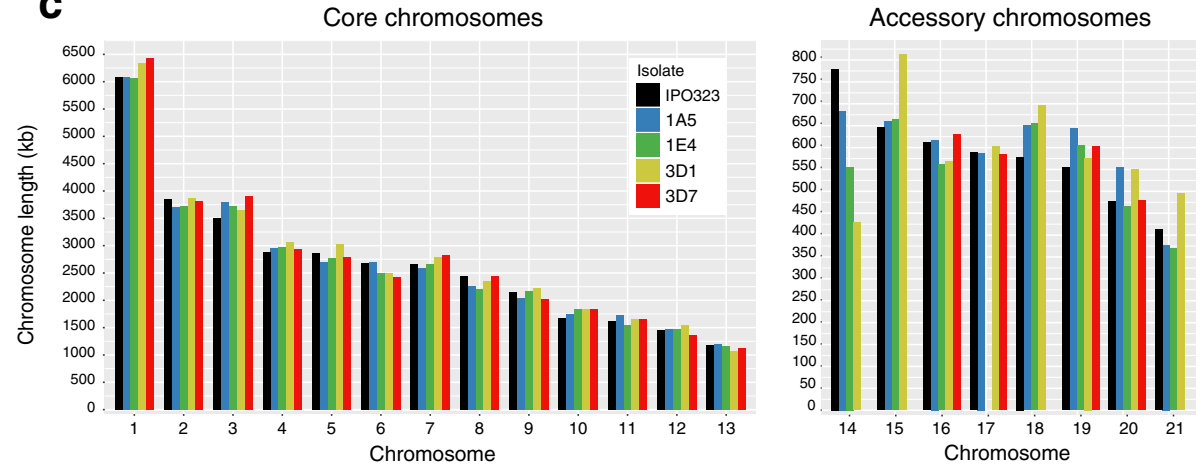

Fig. 1 Comparison of complete genome assemblies of five Zymoseptoria tritici strains used to construct the pangenome. a Maximum likelihood phylogeny of the isolates based on a genome-wide single nucleotide polymorphism (SNP) matrix. The scale refers to the proportion of total SNPs included in the analysis. $\mathbf{b}$ Principal component analysis based on a genome-wide SNP matrix. The variance explained by each of the two axes is shown in parentheses. c Lengths of completely assembled core and accessory chromosomes are shown in separate panels 


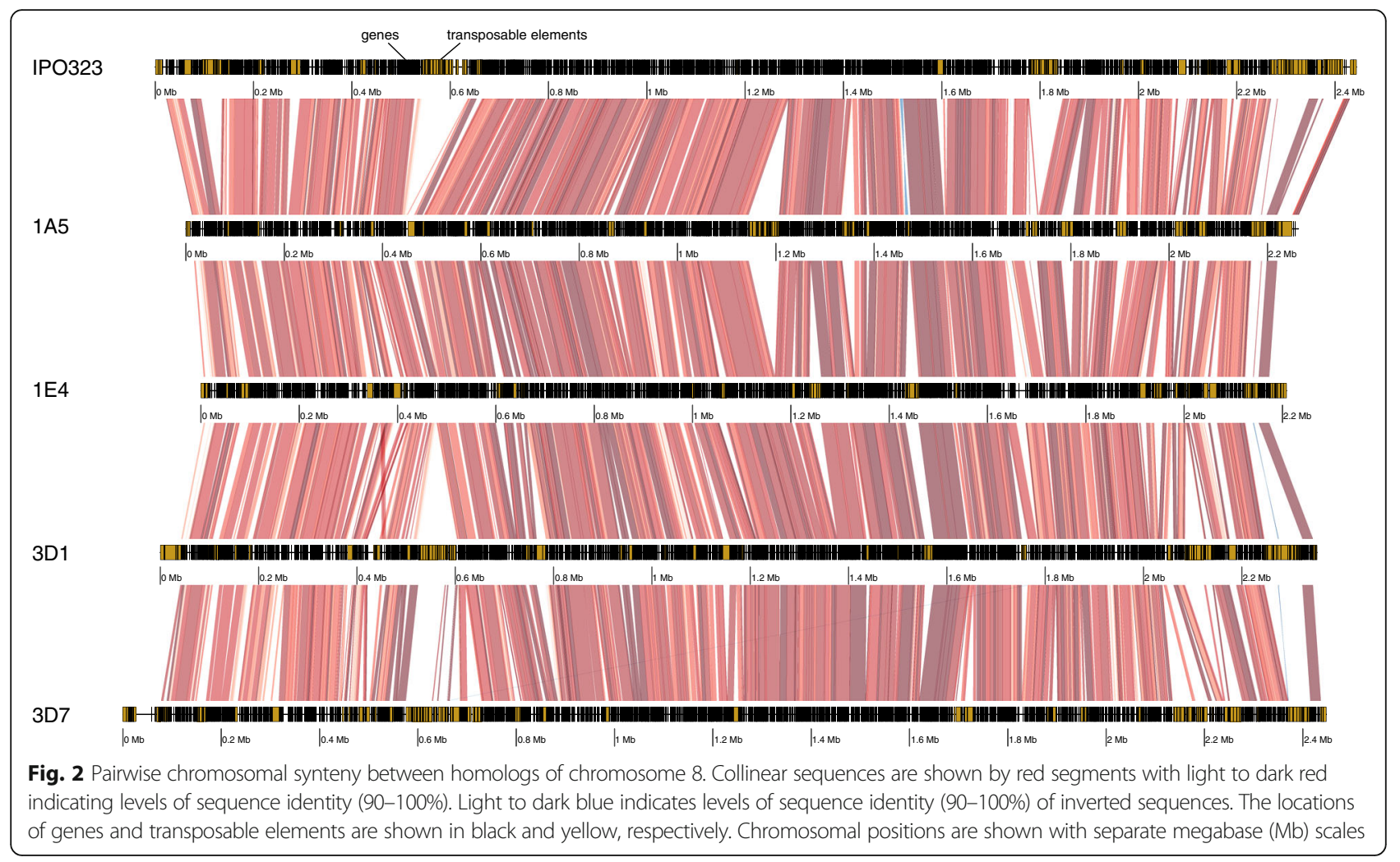

course of a complete wheat infection cycle. To ensure that functional genes were consistently identified across all genomes, we used matches of IPO323 proteins against all other genomes as additional evidence during gene prediction.

The total number of encoded proteins identified in each of the five genomes ranged from 11,737 to 12,092 (Table 1). We evaluated the completeness of both the genome assemblies and the quality of the gene predictions by performing BUSCO analyses on the predicted proteins. We found that all genome annotations were highly complete, ranging from $96.8 \%$ to $98.3 \%$ (Additional file 2: Table S2). We identified a similar number of conserved proteins in each isolate (7353 to 7439). The number of proteins predicted to be secreted (secretome) ranged from 932 to 1059 among isolates (Table 1, Additional file 3: Table S3). We analyzed the set of proteins likely acting as effectors in host interactions based on predictions from EffectorP [50]. We found a total of 221 candidate effectors in the genome of IPO323 and a range of 286-330 candidate effectors among the four Swiss isolates 1A5, 1E4, 3D1, and 3D7 (Table 1).

\section{Pangenome analyses of core and accessory gene sets}

In order to assess shared protein functions among isolates, we constructed a pangenome by clustering the protein sets encoded by each of the genomes of isolates 1A5, 1E4, 3D1, 3D7, and IPO323. The thresholds used at the protein clustering stage affected the number of detected homologs. We conservatively considered proteins with more than $60 \%$ length and more than $75 \%$ sequence similarity as homologs. Decreasing the thresholds did not meaningfully reduce the size of the pangenome, whereas increasing the thresholds increased the pangenome size by approximately $20 \%$ (Additional file 3 : Table S3). We identified a total of 15,749 non-redundant proteins, of which 9149 (58.1\%) were shared among all five analyzed genomes (Fig. 3a, Additional file 4: Table S4). We defined this set of proteins to be encoded by the core genome. The accessory genome of all strains combined encoded 6600 (41.9\%) non-redundant proteins. The accessory genome encoded 3377 (21.4\%) proteins shared by at least two isolates and 3223 (20.4\%) singleton proteins found in only one isolate (Fig. 3b). IPO323 and 3D7 had a higher proportion of singleton proteins, with 1006 and 859 proteins, respectively (Fig. 3a). Isolates 1A5, $1 \mathrm{E} 4$, and 3D1 had comparable numbers of singleton proteins (411-483). The number of singleton proteins reflected the genetic distances among the isolates as shown by the principal component analysis (Fig. 1b).

Among the 15,749 proteins encoded by the pangenome of $Z$. tritici, only 76 proteins had a paralog. This low number of paralogs may be a consequence of efficient repeat-induced point (RIP) mutations introduced into any recently duplicated sequence [51]. Most paralogs originated from simple duplication events 
Table 1 Genome assembly and annotation statistics of the five complete Zymoseptoria tritici genomes

\begin{tabular}{|c|c|c|c|c|c|}
\hline Genomes assembly & 1A5 & 1E4 & 3D1 & $3 \mathrm{D} 7$ & IPO323 \\
\hline Genome size, Mb & 39.7 & 38.6 & 40.7 & 37.9 & 39.7 \\
\hline Chromosomes, $\mathrm{n}$ & 21 & 20 & 21 & 17 & 21 \\
\hline \multicolumn{6}{|l|}{ Gene annotation } \\
\hline Predicted genes & 12,092 & 12,033 & 12,006 & 11,737 & 11,839 \\
\hline Average gene length, bp & 1520.2 & 1524.2 & 1514.1 & 1502.6 & 1620.9 \\
\hline Average protein length, aa & 467.5 & 468.3 & 466.4 & 457.2 & 487.8 \\
\hline Number of exons & 29,716 & 30,015 & 29,033 & 30,399 & 30,068 \\
\hline Average exons per gene & 2.46 & 2.49 & 2.42 & 2.59 & 2.54 \\
\hline Average exon length, bp & 570.7 & 563.3 & 579.8 & 530.7 & 575.2 \\
\hline Number of introns & 17,628 & 17,984 & 17,027 & 18,663 & 18,226 \\
\hline Average introns per gene & 2.18 & 2.21 & 2.15 & 2.29 & 2.54 \\
\hline Average intron length, bp & 80.7 & 79.7 & 79 & 80.6 & 91.6 \\
\hline Genes with introns & 8099 & 8129 & 7925 & 8142 & 8044 \\
\hline Gene density, $\mathrm{Mb}^{-1}$ & 304.6 & 311.7 & 294.9 & 309.7 & 298.3 \\
\hline \multicolumn{6}{|l|}{ Protein functions } \\
\hline Proteins with Pfam domain & 7403 & 7439 & 7431 & 7353 & 7375 \\
\hline Secreted protein & 1025 & 1059 & 1014 & 1034 & 932 \\
\hline Predicted effectors ${ }^{a}$ & 309 & 286 & 296 & 330 & 221 \\
\hline
\end{tabular}

${ }^{\mathrm{a}}$ Effectors were predicted using the software EffectorP [50]

(Additional file 5: Table S5). Three of the paralogs (Cluster_002657, Cluster_007468, and Cluster_009805) encoded candidate effectors. The most expanded para$\log$ families were Cluster_001114, Cluster_000001, Cluster_000201, Cluster_000202, and Cluster_000200, and most paralog members were found in the 1E4 and $1 \mathrm{~A} 5$ genomes with up to 13 and 24 copies, respectively (Additional file 5: Table S5).

Differences in encoded proteins among isolates can stem from several factors, including gene gains and losses, as well as segregating loss-of-function (LOF) mutations. We evaluated IPO323 proteins for which we found no pangenome homolog in either one of the four other isolates. Thus, such genes were classified as accessory or singleton proteins. We mapped Illumina whole genome sequencing data from each of the four other isolates to the genome of IPO323 and analyzed sequence coverage. We found that $31-35 \%$ of the genes for which an isolate was not predicted to have a functional protein lacked sequence coverage (Additional file 6: Table S6, Additional file 7: Figure S1). We found that an additional $46-51 \%$ had at least partial sequence coverage but contained LOF mutation causing protein truncations (Additional file 6: Table S6). Finally, we mapped RNAseq data of each isolate to the genome of IPO323 and quantified expression levels of the accessory genes. We found that, overall, $80-83 \%$ of all genes lacked transcription over the course of an infection (Additional file 6: Table S6). In summary, isolates lacked accessory proteins due to a combination of gene deletions and LOF mutations. Additional factors may be related to pseudogenization, as suggested by a lack of transcription. In summary, these factors accounted for a total of approximately $90 \%$ of accessory genes in the pangenome (Additional file 6: Table S6).

\section{Structure and function of the pangenome}

We found that accessory and singleton genes were frequently clustered into blocks of 2-23 genes in the IPO323 genome (Fig. 3c). The degree of clustering was significantly higher than expected from a random distribution of gene localizations (Fisher exact test, $P<0.0001$ ). In the IPO323 genome, singleton genes were closest to transposable elements followed by accessory and core genes (Fig. 4). As an evolutionary response to prevent transposable element proliferation, regions rich in transposable elements can be silenced through histone lysine methylation. Specific methlyations are associated with euchromatin (H3K4me2), obligate (H3K9me3), and facultative (H3K27me3) heterochromatin regions. All pangenome gene categories were at similar distances to euchromatin regions (Fig. 4). Mirroring the association with transposable elements, singleton genes were closer to regions of obligate heterochromatin. In contrast, core genes were closer to regions of facultative heterochromatin (Fig. 4). In 


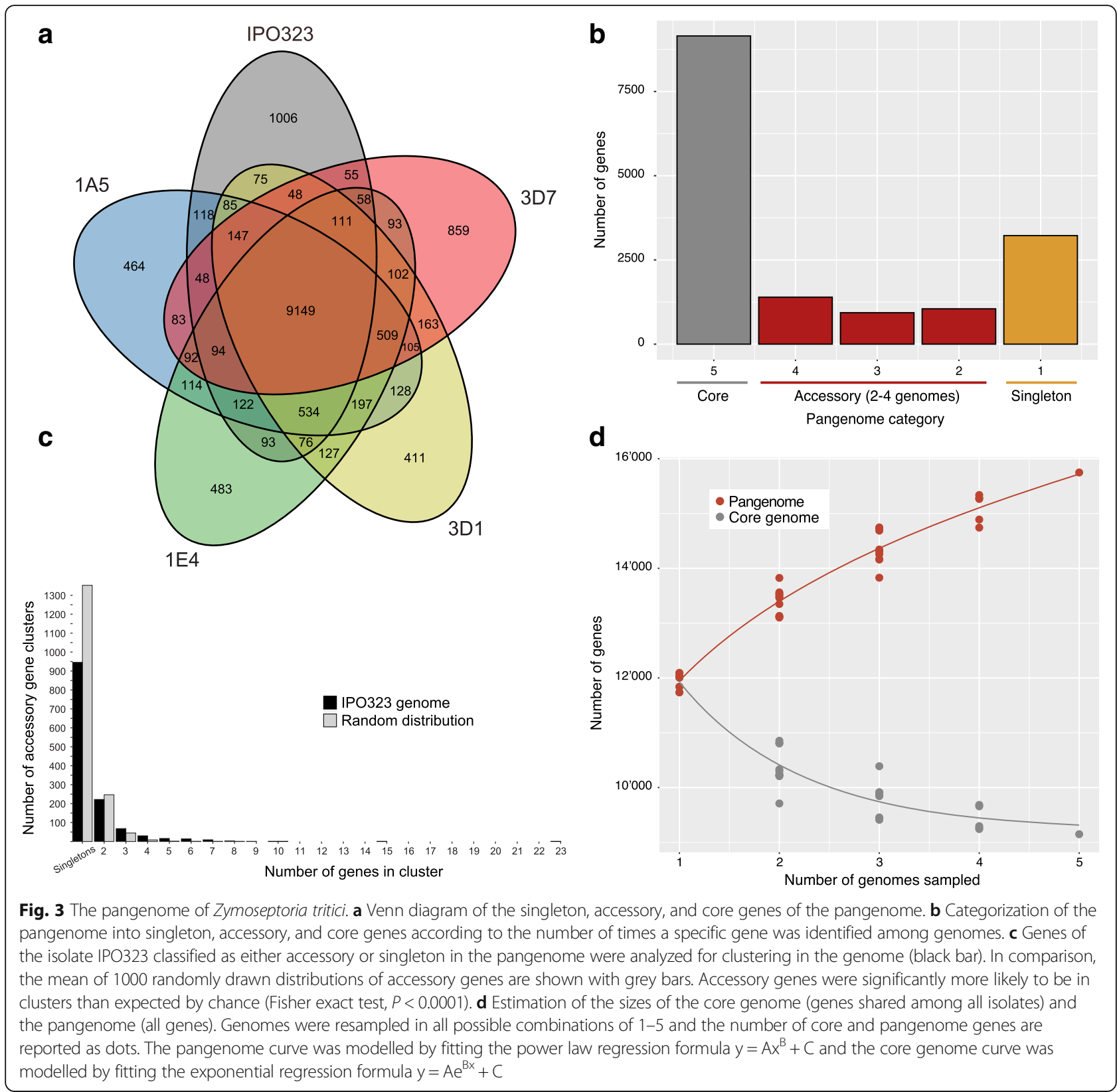

the $Z$. tritici genome, facultative heterochromatin was mostly associated with subtelomeric regions, while obligate heterochromatin was enriched in regions rich in transposable elements.

We estimated the total size of the core and pangenome by resampling 1-5 genomes and reporting the size of the core and pangenome. We found that the core genome likely stabilizes at approximately 9000 core genes (Fig. 3d). However, the number of accessory genes discovered with each additional genome did not stabilize and the pangenome size increased almost linearly with the number of genomes (Fig. 3d). Thus, the complete pangenome of the species is likely substantially larger than estimated from these five genomes.

We analyzed three categories of genes constituting the pangenome of $Z$. tritici, namely core genes shared among all five genomes, accessory genes found in 2-4 genomes, and singleton genes found only in a single genome. We defined these categories to approximate the gene frequencies in the species and identify specific characteristics of subsets of the pangenome. The average amino acid sequence length was significantly different between the three categories, with core genes encoding on average the longest amino acid sequences 


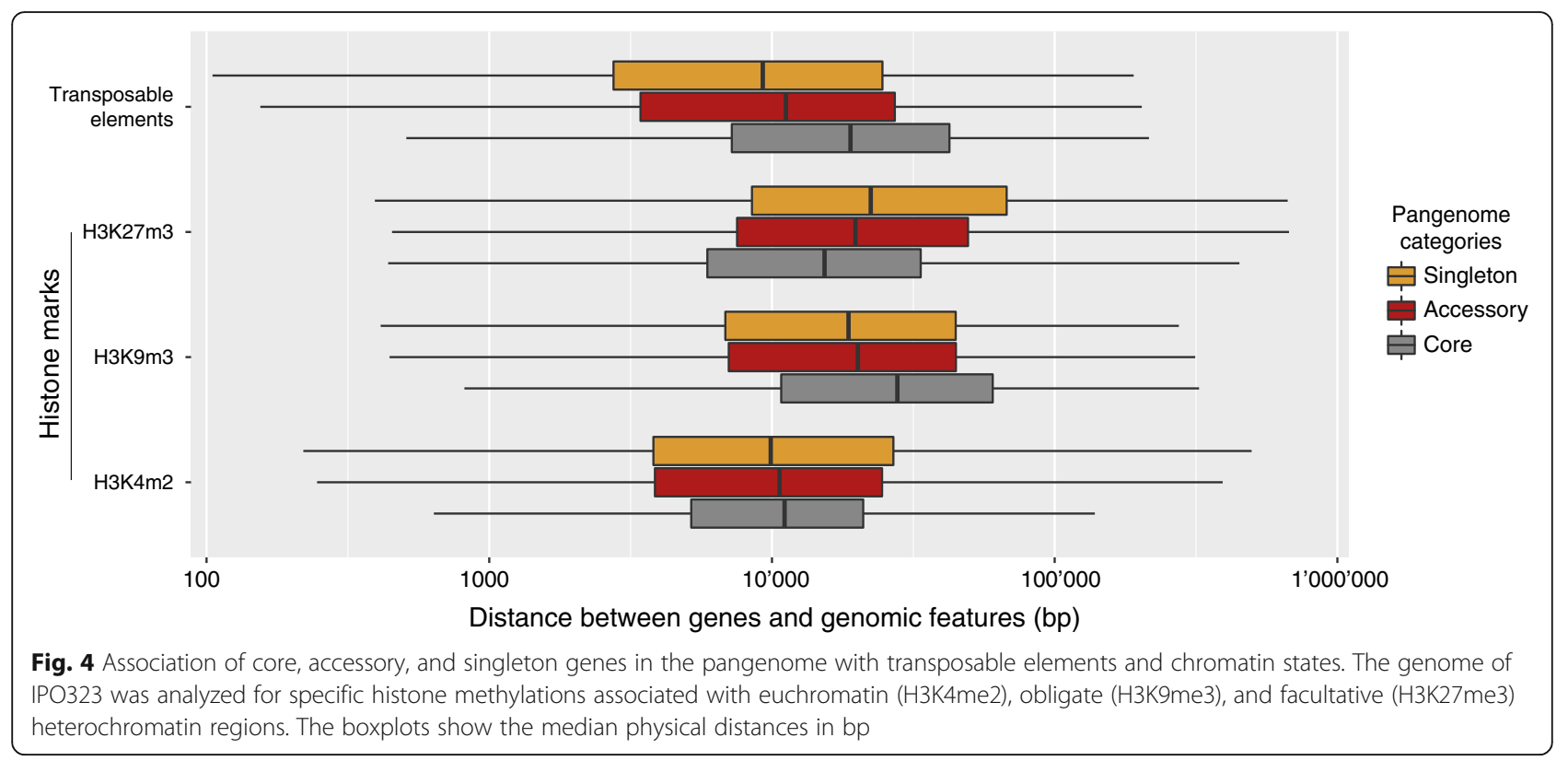

(Kruskal-Wallis test, $P<0.0001$, Fig. 5a). Among core genes, $67 \%$ encoded a conserved protein domain. The percentage decreased to $32 \%$ and $20 \%$ for accessory and singleton genes (Fig. 5b). Pathogen genomes encode a large number of secreted proteins with roles in host interactions. We found that both secreted proteins and effector candidates were significantly enriched among proteins encoded by accessory and singleton genes, with the highest proportion found in accessory genes (Fig. 5c, d). We identified a total of 153 core effector candidates shared among all five genomes as well as 232 accessory and 120 singleton effector candidates (Fig. 5e). The genomes of IPO323 and 3D7 encoded the largest number of singletons with 44 and 35 effector candidates, respectively. Levels of gene transcription also varied among gene categories with core genes showing the highest and singletons showing the lowest median transcription levels during infection of wheat (Fig. 5f).

We analyzed whether different gene categories of the pangenome encoded distinct protein functions. For this, we performed gene ontology (GO) term enrichment analyses. We found that core genes were significantly enriched in housekeeping genes including basic cellular functions and development. Core genes were also significantly enriched in genes involved in general metabolism functions such as lipid, sugar or amino acid metabolism, biosynthesis and cell cycle (Additional file 8: Figure S2, Additional file 9: Figure S3 and Additional file 10: Figure S4). We found distinct GO terms to be enriched in accessory and singleton gene categories. Accessory genes were significantly enriched in functions such as transmembrane transporter activity (Additional file 10: Figure S4). Moreover, we found enrichment in cellular membrane localization (Additional file 8: Figure S2). We also identified an enrichment in zinc ion binding functions and protein kinase activity, which suggests that the encoded proteins play a role in signaling and transcriptional regulation. Singleton genes were most significantly enriched in functions related to DNA integration processes (Additional file 8: Figure S2). Such functions often originate from the insertion of transposable elements in protein coding genes. We also found significantly enriched functions related to host adaptation, including defense responses, chitin catabolic processes, as well as hydrolase and cysteine-type peptidase activities.

\section{Pangenome characteristics in a worldwide collection of Z. tritici}

We investigated whether the distinct properties of the pangenome gene categories were representative of specieswide patterns in gene presence/absence variation and LOF mutations. For this, we analyzed polymorphism detected in a worldwide collection of $123 \mathrm{Z}$. tritici isolates. We mapped Illumina short-read sequencing data of all isolates to each of the five completely assembled genomes (Additional file 11: Table S7). Taking the 15,749 non-redundant genes of the pangenome as a reference, we determined, for all isolates, whether the pangenome genes were present and functional (lacking frameshift mutations). We found that each of the 123 isolates encoded between 11,683 and 12,684 functional genes (Fig. 6a). Non-synonymous nucleotide diversity was significantly lower in core genes than in accessory genes (Kruskal-Wallis test $P<0.0001$; Fig. 6b, Additional file 12: Table S8). Next, we analyzed copy 

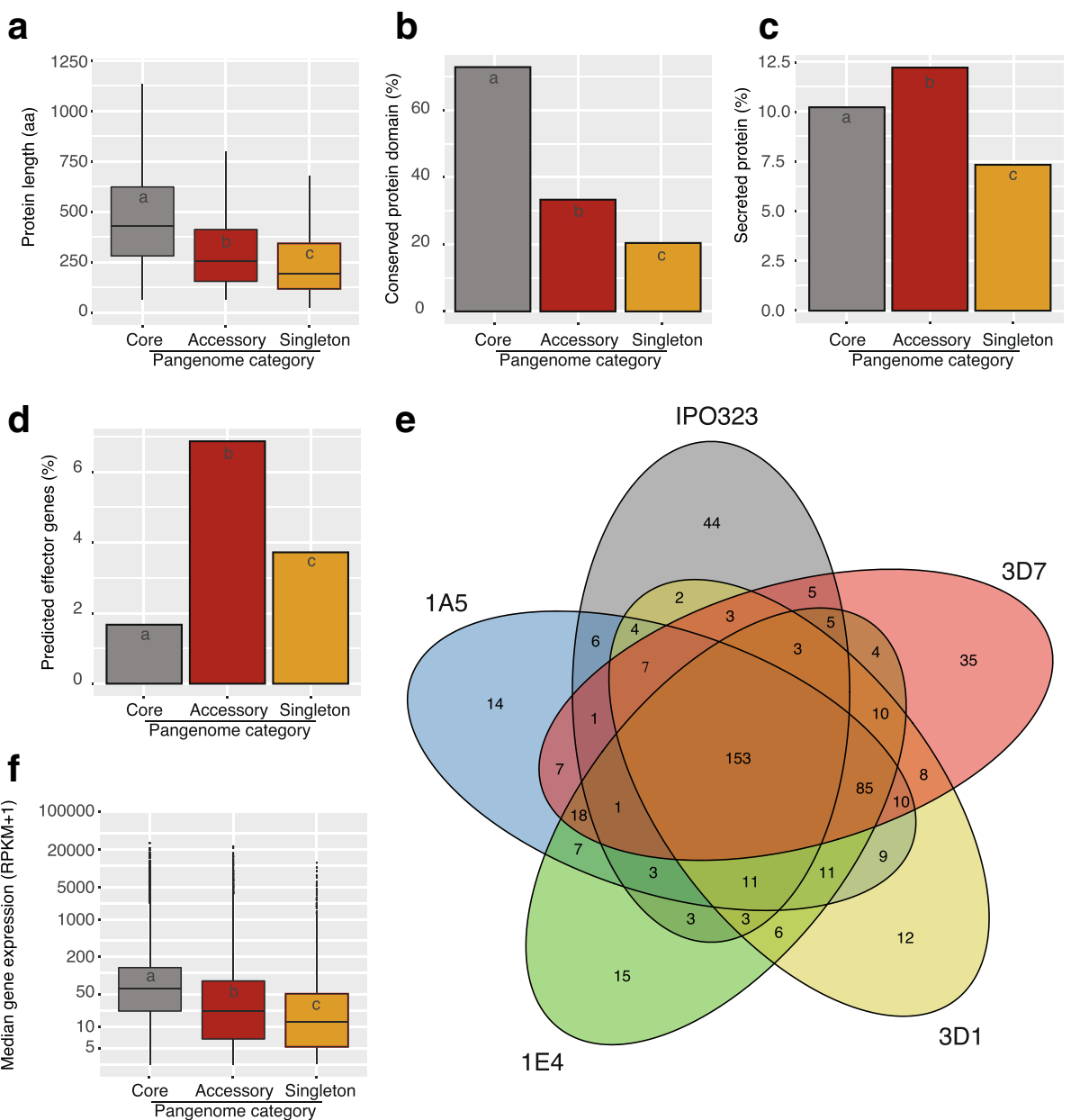

Fig. 5 Functional analyses of the Zymoseptoria tritici pangenome. a Lengths of the encoded proteins by core, accessory, and singleton genes of the pangenome (different letters are $P<0.0001$, Kruskal-Wallis test). $\mathbf{b}$ Percentage of proteins with a conserved protein domain (different letters are $P<0.0001$, Fisher exact test). $\mathbf{c}, \mathbf{d}$ Percentage of genes encoding secreted proteins and candidate effectors, respectively (different letters are $P<0.0001$, Fisher exact test). e Venn diagram of shared and unique effector candidates in the pangenome of isolates 1A5, 1E4, 3D1, 3D7, and IPO323 (reference genome). $\mathbf{f}$ Median expression levels of pangenome genes (different letters are $P<0.0001$, Kruskal-Wallis test)

number variation based on mapped read depth using CNVnator. Among all 123 isolates and all 15,749 nonredundant genes of the pangenome, we detected a total of 113,350 individual gene deletions. A total of 4556 loci showed evidence for a complete gene deletion in at least one isolate. We found strong differences in the rate of gene deletions between the core and accessory genome (Fig. 6c). Only $7.8 \%$ of the core genome loci showed evidence for deletions among the global collection of isolates, but $55.3 \%$ and $61.2 \%$ of the accessory and singleton genome loci, respectively, showed evidence for deletions. The majority (52\%) of the gene deletion loci had a low deletion frequency among isolates $(<10 \%)$. Core genome loci had the lowest deletion frequencies with an average of $1.2 \%$ (Fig. 5a). Accessory and singleton genes showed higher frequencies of individual gene deletions, with averaged frequencies of $9.6 \%$ and $16.8 \%$, respectively.
Pseudogenization often precedes gene loss and could generate additional variation in gene content within the species. We investigated two dominant causes of pseudogenization, namely premature stop codons and frameshift mutations. To identify these two types of LOF mutations, we analyzed single nucleotide polymorphisms (SNPs) and small indels segregating among the 123 isolates. As for the coverage analyses above, we called polymorphisms independently using reads mapped against each of the five completely assembled genomes (Additional file 6: Table S6). We found LOF mutations in a total of 8669 genes spanning all gene categories of the pangenome. Overall, $45.4 \%$ of the core genes, $68.6 \%$ of the accessory genes, and $68.2 \%$ of the singleton genes had at least one LOF mutation in at least one isolate. The frequency of LOF was significantly higher in accessory genes compared to core genes (Kruskal-Wallis test, $P<0.0001$; Fig. 6d). 

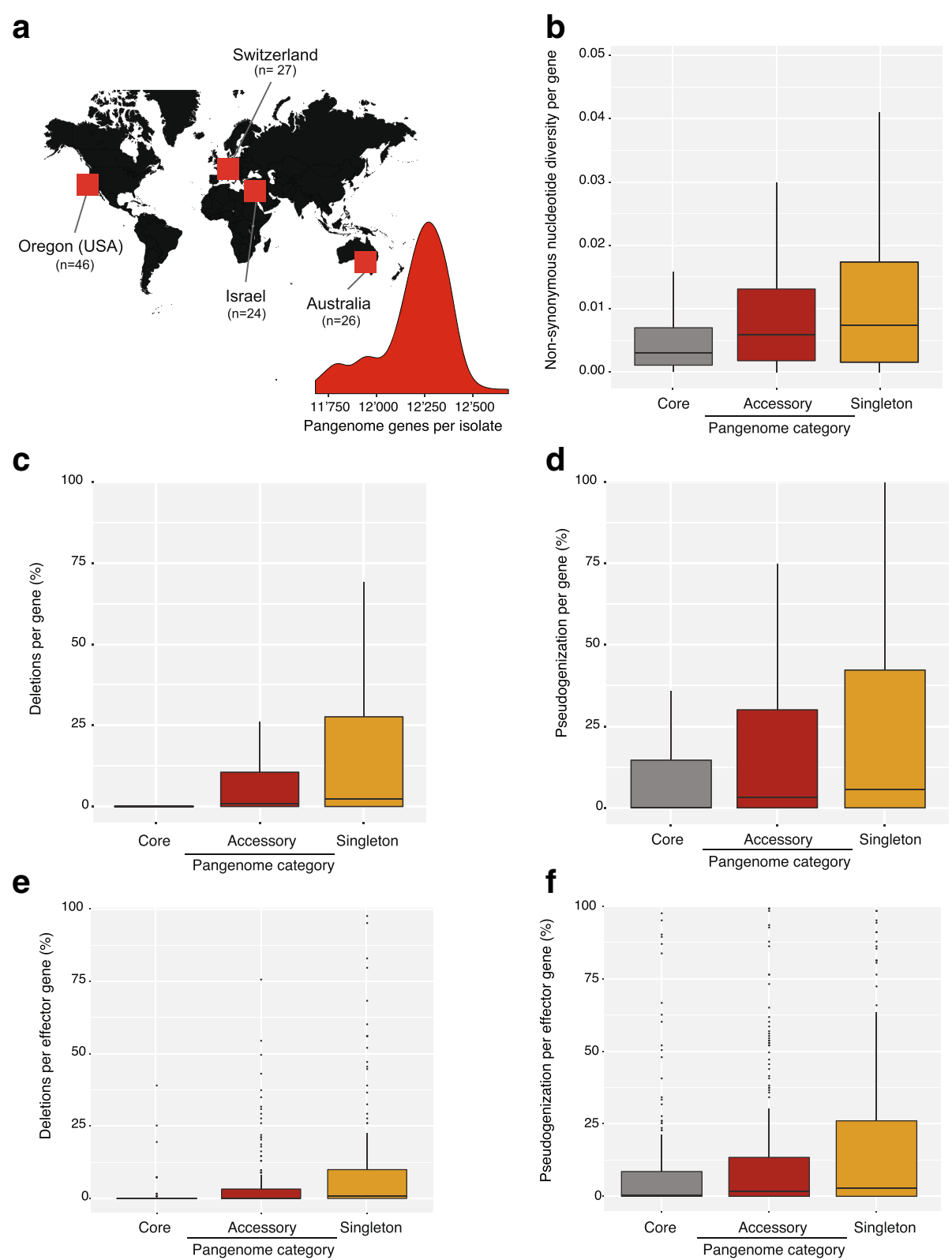

Fig. 6 Population genomic analyses of different pangenome gene categories. a Illumina whole-genome sequencing data of 123 Zymoseptoria tritici isolates covering the worldwide distribution range was analyzed for polymorphism in core, accessory, and singleton genes of the pangenome. The distribution shows the number of pangenome genes detected in each isolate. Gene presence was defined as showing no evidence for deletion (based on sequence coverage) and no loss-of-function (LOF) mutations (based on variant calling). b Non-synonymous nucleotide diversity ( $\pi_{n}$ ) per gene. $\mathbf{c}$ Percentage of isolates showing evidence for a gene deletion. $\mathbf{d}$ Percentage of isolates showing evidence for LOF mutations. e Percentage of isolates showing evidence for an effector gene deletion. $\mathbf{f}$ Percentage of isolates showing evidence for LOF mutations in effector genes

\section{Pangenome analyses of effector genes}

In $Z$. tritici, two major effector genes were identified using association mapping [42, 43]. The first effector $Z t \_8 \_609$ caused avirulence on the wheat cultivar Toronit and was found to segregate a presence-absence polymorphism within the species. Consistent with previous analyses showing that $Z t \_8 \_609$ was missing in 1A5, 1E4, 3D1, and $3 \mathrm{D} 7$ [42], the gene was identified as a singleton in IPO323
(Additional file 3: Table S3). The effector AvrStb6 causing avirulence on cultivars carrying the resistance gene Stb6 was known to be missing in the annotation of IPO323 [43]. However, the gene was recovered and clustered at the protein level in the annotations of 1A5, 1E4, 3D1, and 3D7 (Additional file 3: Table S3).

We more broadly analyzed gene deletions and LOF mutations affecting genes that encoded candidate 
effectors. The pangenome analyses identified a total of 591 candidate effectors, of which 153 belonged to the set of core genes (Fig. 5e). We analyzed the polymorphism of candidate effector genes in the collection of 123 worldwide isolates. We observed a similar pattern for effector genes than for all gene categories combined. Effector genes showed much stronger conservation within the species if they were core genes. Accessory and singleton effector genes had both higher rates of gene deletions and LOF mutations (Fig. 6e, f). We identified a total of 134 effector genes without any evidence for gene deletion or LOF mutations in any of worldwide isolates. Among these highly conserved effectors, 8 were also conserved at the amino acid level and showed strong up-regulation during plant colonization. This small proportion of highly conserved effectors likely play an essential role in pathogenicity.

\section{Discussion}

We constructed a pangenome for the highly polymorphic wheat pathogen Z. tritici based on five completely assembled and independently annotated isolates. We identified a much larger gene repertoire than previously known from the reference genome alone. The core and the accessory genome showed distinct functional, structural and regulatory characteristics. We confirmed the distinct properties of the core and accessory genome by analyzing sequence data of a worldwide collection of 123 additional isolates. Accessory genes were more likely to be polymorphic, contain frameshift mutations and more likely to be deleted compared to core genes. We identified eight highly conserved effector candidates within the species that may play an essential role in pathogenesis.

\section{A pangenome to approximate the complete gene repertoire of the species}

Many fungal species vary considerably in genome size among closely related species and show chromosome length polymorphism within species [34, 52]. Early evidence for chromosome length polymorphism in $Z$. tritici was found based on gel electrophoresis [46]. A recent population genomics study showed that $Z$. tritici populations harbor extensive gene deletion polymorphisms affecting $15 \%$ of the genes identified in the reference genome [53]. A comparison of two completely assembled genomes showed that either strain harbored a similar number of additional genes compared to the other strain [14]. Our pangenome construction confirmed that $Z$. tritici harbors a significantly larger gene repertoire than known from the reference genome alone. The gene space of the reference genome IPO323 was estimated to be composed of 11,795 complete genes [54] and our minimum estimate of the gene repertoire of the pangenome was 15,749 genes.

The total number of distinct genes encoded in Z. tritici genomes is likely to be substantially higher for two reasons. First, our estimates were based on four Swiss and one Dutch isolates, which are unlikely to fully represent the global polymorphism of the species. Nevertheless, single field populations of $Z$. tritici harbor a substantial portion $(\sim 90 \%)$ of the total genetic variation within the species due to high effective population sizes and gene flow [38]. Second, our resampling estimates of the total gene content did not stabilize at a plateau with five genomes.

The open nature of the $Z$. tritici pangenome means that a substantial number of additional genes will be identified by assembling additional genomes. In particular, analyzing genomes from the genetically diverse populations in the Middle East and Fertile Crescent, where the pathogen was co-domesticated with wheat, is likely to drastically increase the size of the pangenome. In contrast to the open pangenome, we obtained a confident estimation of the core genome size. Our resampling showed that including additional genomes is unlikely to substantially reduce the set of genes shared within the species. We confirmed this prediction by analyzing the gene content of 123 worldwide isolates and indeed found that more than $98 \%$ of all genes defined as core genes were present in all isolates. Core genes also shared a number of properties indicative of their essential role for the organism, and were significantly enriched among others in functions related to basic cellular processes and growth. Loss of genes encoding essential functions would be strongly deleterious.

In contrast to the core genome, the accessory genome was enriched in genes playing important roles in pathogenicity. Plant pathogen genomes encode effectors that can manipulate host immune defenses and largely determine the outcome of infections. Thus, comprehensive knowledge of effector gene loci is crucial to gain insights into the infection process. We identified hundreds of effector genes in the newly assembled genomes of isolates $1 \mathrm{~A} 5,1 \mathrm{E} 4$, and 3D1, which were not present in the previously assembled genomes of IPO323 and 3D7 $[14,45,54]$. We identified 250 effector genes that were lacking in the IPO323 reference genome, which is greater than the total number of effectors encoded by the IPO323 genome (221). However, differences in effector gene predictions may also be influenced by the annotation strategy [55]. Predictions for IPO323 were performed using evidence for gene models extracted from transcriptomic data of $Z$. tritici growing on culture medium and during the earliest infection stages [54]. The newly assembled genomes were annotated using transcriptomic evidence across the entire infection cycle 
[56]. This difference may indeed be important because the expression of many effector genes coincides with the development of large scale lesions and the onset of pathogen reproduction [56]. An example of a missed major avirulence effector is AvrStb6 [43]. The effector gene was missing from genome annotations of the IPO323 reference genome until evidence for the gene was identified using more comprehensive transcriptomic data. In contrast, the second known avirulence effector, Zt_8_609, was properly annotated in the reference genome IPO323, but the gene is deleted in all newly assembled genomes [42]. Our pangenome analyses significantly expanded the effector gene repertoire of $Z$. tritici and provides an essential resource to identify yet unknown avirulence factors.

\section{Birth and death of genes in the pangenome}

The pangenome constructed for $Z$. tritici comprised $42 \%$ accessory genes (i.e., not fixed within the species). A more expansive analysis of the pangenome covering more genomes would increase this proportion even further. The extent of the accessory genome raises significant questions with regard to the evolutionary origin of this polymorphism, its role in adaptive evolution, and the trajectory of potentially redundant gene functions. A substantial proportion of the $Z$. tritici accessory genome was generated through LOF mutations segregating among isolates. These mutations were predominantly deleterious frameshifts and early stop codons causing protein truncation. Even if expressed, truncated proteins are unlikely to perform an identical biological function compared to the fulllength protein variant and, thus, contribute to functional variation among isolates. Presence-absence polymorphism of genes accounted for slightly less accessory genome polymorphism than LOF mutations. Our analyses of a worldwide collection of isolates confirmed the major characteristics of the pangenome. In addition to higher frequencies of gene deletions, accessory genes were more likely to segregate LOF mutations. We also found that accessory genes had higher non-synonymous nucleotide diversity, which is indicative of relaxed selection caused by functional redundancy.

In a previous analysis of $Z$. tritici, 1623 segregating gene presence-absence polymorphisms were investigated for their evolutionary origins [53]. Interestingly, approximately two-thirds of these polymorphisms were generated by deletions of genes that were shared among closely related species. One-third of the presenceabsence polymorphisms affected genes lacking homologs. Therefore, these genes were likely recently gained and had not yet reached fixation within the species. In principle, loss of essential gene functions should be under strong negative selection in haploid organisms [57]. Given the size of the $Z$. tritici accessory genome, a substantial fraction of all $Z$. tritici genes are likely functionally redundant or have no specific function (i.e., the genes are dispensable).

Functional redundancy is unlikely to have evolved by individual gene duplications or whole genome duplications. In fact, both our analysis of the pangenome and previous analyses of the reference genome IPO323 revealed little evidence for paralogy [45]. A likely explanation for the lack of paralogs is that the genome of $Z$. tritici encodes the machinery for the genomic defense mechanism RIP. RIP introduces random mutations in any near identical sequence copy in the genome [58]. RIP primarily evolved as a defense against the activity of transposable elements, but it also constrains evolution by gene duplication [51]. Thus, if gene dispensability evolved as a consequence of redundancy, genes performing similar functions are unlikely to be related by common descent. Functional redundancy in absence of paralogy could explain why many effector gene knockouts have no detectable impact on pathogenicity $[59,60]$. Importantly, functional redundancy in pathogen effectors can serve the evolutionary purpose of a 'bedhedging' strategy. A change in the environment, such as the introduction of a host detecting a specific effector, will impose strong selection on the pathogen population to eliminate this effector. Thus, a pathogen population that is highly heterogeneous in effector complements can recover from such selection by favoring non-recognized effectors that converge on the same function as a recognized effector [61].

In addition to redundancy, environmental specificity or local adaptation can be a driver of gene dispensability and, ultimately, gene loss [62]. Some accessory genes could play a role under specific environmental conditions and, thus, contribute to local adaptation. As for functional redundancy, the strongest candidate genes for such environmental specificity would be effector genes that evolved to promote infection on specific host genotypes. Wheat cultivars are genetically highly diverse and encode at least 21 distinct resistance loci against the pathogen [63]. It is thought that most wheat resistance loci are matched by a specific pathogen effector. A pathogen expressing the matching effector gene is unable to colonize the host. Thus, geographic heterogeneity in the deployment of wheat genotypes would favor specific effector gene losses in specific regions. Evidence for such an adaptive gene loss was found for a $Z$. tritici effector gene [42].

\section{Conclusions}

Z. tritici populations maintained very significant levels of standing genetic variation as indicated by the speed of adaptive evolution and decay in linkage disequilibrium $[37,39,40]$. Effective population size is an important determinant of pangenome sizes [64]. In absence of selection, large populations should maintain pangenomes by 
preventing accessory genes to be lost from the gene pool by random drift. A constraint in the expansion of the pangenome likely entails the necessity for homologous chromosome pairing during meiosis. As the Z. tritici accessory genome was associated with substantial structural variations, some accessory regions may already show reduced recombination rates. In sufficiently large populations, at least a fraction of the accessory genes could have recently evolved from non-coding DNA. Indeed, de novo gene evolution is common in the model fungus Saccharomyces cerevisiae and other eukaryotes $[5,65]$. At an early stage, de novo genes would likely have no function and show weak expression, characteristics shared by many genes of the accessory genome. Retracing the evolutionary origin of accessory genes will provide significant insights into the emergence of highly polymorphic pangenomes and their role in adaptive evolution [23, 27, 28, 66, 67]. The analyses of eukaryotic pangenomes will elucidate how major evolutionary novelty arises within species.

\section{Methods \\ High molecular-weight DNA extraction and single molecule real-time (SMRT) sequencing}

The isolates ST99CH_1A5, ST99CH_1E4, and ST99CH_3D1 (abbreviated here as 1A5, 1E4, and 3D1, respectively) were sampled from two Swiss wheat fields in 1999 [68]. In order to extract high molecular-weight DNA, we used the modified version of the cetyltrimethylammonium bromide DNA extraction protocol described in Plissonneau et al. [14]. In brief, fungal spores were harvested after 5-6 days in liquid yeast sucrose broth and lyophilized. Approximately 60-100 mg of lyophilized spores were crushed with a mortar and pestle. From a phenol-chloroform-isoamyl alcohol solution, the supernatant was transferred and centrifuged. The pellet was resuspended in phenol-chloroformisoamyl alcohol and repeated twice. For each isolate, PacBio SMRTbell libraries were prepared using $>15 \mu \mathrm{g}$ of high molecular-weight DNA. Sequencing was performed using P6/C4 chemistry on a PacBio RSII instrument at the Functional Genomics Center, Zurich, Switzerland.

\section{Complete genome assemblies}

We used the pipeline developed previously for the Z $Z$. tritici isolate 3D7 [14]. In summary, raw sequencing reads were assembled using HGAP v 3.0 included in the SMRTanalysis suite (version 2.3.0, patch 3) [21]. All settings were at default, with the exception of the minimum seed read length to initiate the self-correction. Different seed read length cut-offs were tested to compensate for preassembly yield. The assembled contigs were polished using Quiver with default settings as implemented in the
SMRTanalysis suite. Problematic contigs were identified by the mapped PacBio read coverage. Contigs were discarded if the mean mapped coverage deviated by more than $1.5 \times$ from the median coverage of all contigs (weighted by length). We previously generated and genotyped crosses of $1 \mathrm{~A} 5 \times 1 \mathrm{E} 4$ and $3 \mathrm{D} 1 \times 3 \mathrm{D} 7$, respectively [49]. Thus, we were able to evaluate the contiguity of contigs using the high-density SNP-based genetic maps. All assembled contigs were uniquely assigned to a single linkage group. The genetic and physical marker orders were highly correlated. Completely assembled chromosomal sequences were quality checked using short read data available for each of the strains (NCBI Short Read Archive accessions SRS383142, SRS383143, and SRS383146). We used the PILON procedure to map Illumina short reads and corrected indels and SNPs detected in the Illumina read alignments [69]. See Plissonneau et al. [14] for more details on the SNP calling, map construction, and assembly validation procedure.

\section{Gene prediction and genome annotation}

To accurately predict the gene space of each newly assembled genome, we used the gene prediction software BRAKER v1.9 [70]. BRAKER combines coding sequence and intron hints based on the mapping of conserved protein sequences and introns identified in RNA-seq data, respectively. We used RNA-seq datasets generated for isolates $1 \mathrm{~A} 5,1 \mathrm{E} 4$, and 3D1 [56], which covered four time points $(7,12,14$, and 28 days after inoculation) over the course of an infection of the susceptible wheat cultivar Drifter. All sequencing datasets were retrieved from the NCBI Short Read Archive under the project accession number SRP077418. Raw RNA-seq reads were quality filtered using Trimmomatic $\mathrm{v} 0.36$ [71] using the following parameters: ILLUMINACLIP:TruSeq3-PE.fa: 2:30:10 LEADING:10 TRAILING:10 SLIDINGWIN DOW:5:10 MINLEN: 50, and then aligned to the corresponding genome assembly using tophat $\mathrm{v}$ 2.0.14 [72] with the following parameters: -min-intron-length 10 -max-intron-length 1000 -mate-inner-dist 180 -matestd-dev 40. To extract predicted intron splice sites, we used the tool bam2hints ( - minintronlen $=10-$ maxintronlen $=1000$-maxgaplen $=9$ ) from the AUGUSTUS v3.2.1 gene prediction software [73]. In addition to intron hints, we used coding sequences annotated in the IPO323 reference genome [54]. For this, we mapped the predicted protein sequences to each assembled genome using exonerate 2.2.0 [74], with the following parameters: - percent 95 -minintronlen $=10$-maxintronlen $=$ $1000-$ model $=$ protein2genome. Finally, intron on coding sequence hints were combined and provided to BRAKER to generate gene predictions for each assembled genome. 


\section{Gene expression analyses}

We quantified gene expression profiles over the course of a wheat infection using RNAseq datasets generated by Palma-Guerrero et al. [56] for isolates 1A5, 1E4, 3D1, and 3D7, and by Rudd et al. [59] for the reference genome isolate IPO323. RNAseq reads were mapped to the corresponding reference genome using tophat $\mathrm{v}$ 2.0.14 [72] using the following parameters: -min-intron-length 10 -max-intron-length 1000 -mate-inner-dist 180 -matestd-dev 40. To obtain read counts for each gene, we used HTSeq v0.7.1 [75] with the parameters $-\mathrm{s}$ reverse $-\mathrm{m}$ union. We calculated normalized counts per million and reads per kilobase of transcript per million mapped reads using the $\mathrm{R}$ package edgeR normalizing read counts individually per isolate across all time points [76].

\section{Annotation of gene functions}

The completeness of the assembled genomes and the corresponding gene annotations was tested using the Ascomycota dataset of BUSCO version 3 [77]. Predicted proteins were assigned to protein families (Pfam) and GO terms using InterProScan 5.18-57 [78]. Secretion signals, as well as cytoplasmic, transmembrane, and extracellular domains, were predicted using a combination of SignalP 4.1 [79], Phobius 1.01 [80], and TMHMM 2.0 [81]. To define the secretome of each isolate, we selected proteins, which were assigned a secretion signal by both SignalP and Phobius. Proteins with predicted transmembrane domains (by Phobius and TMHMM), an extracellular domain or a cytoplasmic domain (by Phobius) were excluded. We analyzed the predicted secretome of each isolate for candidate effector genes using EffectorP [50].

\section{Pangenome analyses}

We constructed the pangenome based on genes identified in the complete genomes of isolates 1A5, 1E4, 3D1, 3D7, and IPO323. We compared each of the five encoded proteomes with BlastP with a cut-off e-value of $1 \times 10^{-5}$ [82]. We then clustered proteins into families based on pairwise alignments using the software SiLiX v1.2.9 [83] and cut-offs set to $75 \%$ identity and $60 \%$ coverage. To extrapolate the core genome and pangenome sizes, we performed a resampling of all possible combinations of 1-5 genomes. We modelled the core genome and pangenome size curves by fitting the power law regression formulas: $\mathrm{y}=\mathrm{Ae}^{\mathrm{Bx}}+\mathrm{C}$ and $\mathrm{y}=\mathrm{Ax}^{\mathrm{B}}+\mathrm{C}$, respectively, in matlab.

Chromosomal synteny between genomes was analyzed using pairwise blastn on repeat masked genomic sequences. Blastn hits were filtered for a minimum identity of $95 \%$, e-values reported as 0 and a minimum alignment length of 2000 bp. Syntenic regions shared between pairs of homologous chromosomes were visualized using the R package genoPlotR [84].

The chromosomal locations of core, accessory and singleton genes were analyzed in the IPO323 genome for the proximity to transposable elements [53]. Chromosomal locations were also analyzed for euchromatin and heterochromatin marks based on previously generated ChIP-seq datasets for the isolate IPO323 [85]. We accessed the datasets from the public gbrowse server (http://ascobase.cgrb.oregonsta te.edu/cgi-bin/gb2/gbrowse/ztritici_public/). For each ChIP-seq sample, we determined significantly enriched domains using the RSEG toolset [86].

\section{Isolate collection and Illumina genome sequencing}

We analyzed Illumina whole-genome sequencing data for $123 \mathrm{Z}$. tritici isolates originating from four worldwide locations (Australia, Israel, Switzerland, and Oregon, USA; Additional file 11: Table S7). Isolates were stored for long-term use in silica at $-80{ }^{\circ} \mathrm{C}$. No clonal genotypes were found among these isolates in previous genetic diversity analyses [87]. A total of 106 isolates were previously sequenced on a Illumina HiSeq 2500 (paired-end, $100 \mathrm{bp}$ read lengths) and raw sequencing data is available on the NCBI Short Read Archive under the BioProject PRJNA178194 and PRJNA327615 [42, 44, 88]. We generated paired-end sequencing data for 19 additional isolates following identical procedures as for the previously available data. We deposited the raw sequencing data on the NCBI Short Read Archive under the BioProject PRJNA327615 (see Additional file 11: Table S7 for more details).

\section{Alignment of Illumina genome sequencing data to complete genomes}

We trimmed the raw sequencing reads for quality and adapter contamination using the software Trimmomatic v 0.36 [71], with the following parameters: ILLUMINA CLIP:TruSeq3-PE.fa:2:30:10 LEADING:10 TRAILING:10 SLIDINGWINDOW:5:10 MINLEN:50. Trimmed reads were aligned separately on each of the five complete genomes with bowtie2 v2.2.29 [89], with the following parameters: sensitive-local -local -phredd33 -X 1000. We identified and marked PCR duplicates with MarkDuplicates of Picard tools v2.6.0 (http://broadin stitute.github.io/picard).

\section{Variant calling and analyses}

We called SNPs and short indels of all 123 worldwide isolates separately against each of the five complete genome assemblies using the Genome Analysis Toolkit (GATK) v 3.7 pipeline [90]. First, we called variants individually for each isolate using the GATK HaplotypeCaller with the following parameters: -nct 4 -emitRefConfidence GVCF 
-variant_index_type LINEAR -variant_index_parameter 128000 -sample_ploidy 1 . The resulting gvcf files were then combined per reference genome and genotyped with the modules CombineGVCFs and GenotypeGVCFs, respectively. The identified SNPs and short indels were filtered for quality using VariationFiltration with the following cut-offs: QD $>20, \mathrm{FS}<0.1$, QUAL $>250, \mathrm{MQ}>30$, $-2<$ BaseQRankSum $<2,-2<$ ReadPosRankSum $<2$, and $-2<$ MQRankSumPos $<2$. We analyzed the predicted impact of each variant using SnpEff v $4.3 \mathrm{~g}$ (with -ud 300) [91]. Variant effects were parsed with SnpSift v4.3 g [92]. To calculate the non-synonymous nucleotide diversity per gene, we extracted non-synonymous SNPs with SnpSift and then used vcftools with the command -haploid -sitepi in vcftools v0.1.14 [93], with the haploid mode patch provided by Julien Y. Dutheil.

\section{Identification of copy number variation}

To detect gene deletions and duplications in the 123 worldwide isolates, we used the software CNVnator [94]. CNVnator uses normalized read depth (RD) to identify copy number variation $(\mathrm{CNV})$. Following standard recommendations, we performed the analyses in bins of $100 \mathrm{bp}$. Raw CNV calls were filtered using the following criteria: for duplications $\mathrm{RD}>2$ and deletions $\mathrm{RD}<0.4$, additional filters were $\mathrm{q} 0<0.5$, length $>500 \mathrm{bp}$, and evalue $<0.05$. We retrieved genes affected by CNVs using bedtools intersect [95]. CNVnator analyses were performed separately for reads mapped to each of the five complete genomes.

\section{GO enrichment analysis}

Enrichment of GO terms of proteins encoded by genes in different categories was tested using hypergeometric tests with a false discovery rate cut-off set to 0.05 . We retained only GO term enrichment results if at least five genes in the genome matched the GO term. All analyses were performed using the R packages GSEABase and GOStats [96].

\section{Genome assembly and annotation data availability}

All complete genome assemblies and corresponding genome annotations were uploaded to the European Nucleotide Archive under accession numbers PRJEB15648, PRJEB20900, and PRJEB20899 for isolates 1A5, 1E4, and 3D1, respectively.

\section{Additional files}

Additional file 1: Table S1. Chromosomal length variation between the five completely assembled genomes of Zymoseptoria tritici. (PDF 58 kb)

Additional file 2: Table S2. Assessment of genome and annotation completeness using BUSCO. (PDF $51 \mathrm{~kb}$ )

Additional file 3: Table S3. Evaluation of protein clustering thresholds in the construction of the pangenome of Zymoseptoria tritici. (PDF $64 \mathrm{~kb}$ )
Additional file 4: Table S4. Protein clusterization and functional annotation of the pangenome of Zymoseptoria tritici. (XLSX 5334 kb)

Additional file 5: Table S5. Paralogs identified in the pangenome of Zymoseptoria tritici. (XLSX $68 \mathrm{~kb})$

Additional file 6: Table S6. Analyses of genes identified in the isolate IPO323 and classified as accessory or singleton genes in the pangenome. (PDF $67 \mathrm{~kb}$ )

Additional file 7: Figure S1. Sequence coverage of Illumina short reads generated from the isolates 3D1, 3D7, 1A5, and 1E4 mapped against the IP0323 genome. For each IPO323 gene, the mean coverage was calculated. The median coverage of all genes of a specific isolate is shown by a vertical bar. (PDF 118 kb)

Additional file 8: Figure S2. Gene ontology enrichment for cellular compartment terms performed for singleton, accessory, and core genes of the Zymoseptoria tritici pangenome. (PDF 164 kb)

Additional file 9: Figure S3. Gene ontology enrichment for biological process terms performed for singleton, accessory, and core genes of the Zymoseptoria tritici pangenome. (PDF $241 \mathrm{~kb}$ )

Additional file 10: Figure S4. Gene ontology enrichment for molecular function terms performed for singleton, accessory, and core genes of the Zymoseptoria tritici pangenome. (PDF $235 \mathrm{~kb}$ )

Additional file 11: Table S7. Whole-genome sequence analyses of the 123 Zymoseptoria tritici isolates included in this study. (XLS 121 kb)

Additional file 12: Table S8. Non-synonymous nucleotide diversity and loss-of-function mutations among 123 Zymoseptoria tritici isolates. (XLSX 6650 kb)

\section{Acknowledgements}

We are grateful for the advice on SMRT sequencing by Andrea Patrignani and Gerrit Kuhn. BA McDonald provided feedback on an earlier version of this manuscript. The sequencing was performed at the Functional Genomics Center Zurich. Marcello Zala provided advice on extraction methods and assistance in the laboratory.

\section{Funding}

The project was supported by the ETH Zurich Research Commission Grant 12-03. CP was supported by an INRA Young Scientist grant. DC is supported by the Swiss National Science Foundation (grant 31003A_173265).

\section{Availability of data and materials}

All complete genome assemblies and corresponding genome annotations were uploaded to the European Nucleotide Archive under accession numbers PRJEB15648, PRJEB20900, and PRJEB20899 for isolates 1A5, 1E4, and 3D1, respectively.

\section{Data deposition}

Genome assemblies and annotation are available at the European Nucleotide Archive under accession numbers PRJEB15648, PRJEB20899, and PRJEB20900.

\section{Authors' contributions}

CP and DC conceived of the study, performed analyses, and wrote the manuscript, FEH contributed analyses tools and edited the manuscript. All authors read and approved the final manuscript.

Ethics approval and consent to participate

Not applicable.

Consent for publication

Not applicable.

Competing interests

The authors declare that they have no competing interests.

\section{Publisher's Note}

Springer Nature remains neutral with regard to jurisdictional claims in published maps and institutional affiliations. 


\section{Author details}

'Plant Pathology, Institute of Integrative Biology, ETH Zurich, 8092 Zurich, Switzerland. ${ }^{2}$ UMR BIOGER, INRA, AgroParisTech, Université Paris-Saclay, Avenue Lucien Bretignières, BP 01, Thiverval-Grignon F-78850, France. ${ }^{3}$ Ecologie Systématique Evolution, Univ. Paris-Sud, AgroParisTech, CNRS, Université Paris-Saclay, 91400 Orsay, France. ${ }^{4}$ Laboratory of Evolutionary Genetics, Institute of Biology, University of Neuchâtel, CH-2000 Neuchâtel, Switzerland.

\section{Received: 15 August 2017 Accepted: 16 November 2017}

Published online: 11 January 2018

\section{References}

1. Coghlan A, Eichler EE, Oliver SG, Paterson AH, Stein L. Chromosome evolution in eukaryotes: a multi-kingdom perspective. Trends Genet. 2005; 21:673-82.

2. Ohno S. Evolution by Gene Duplication. New York, NY: Springer; 1970.

3. Zhang J. Evolution by gene duplication: an update. Trends Ecol Evol. 2003; 18:292-8

4. Fitzpatrick DA. Horizontal gene transfer in fungi. FEMS Microbiol Lett. 2012; 329:1-8.

5. Tautz D, Domazet-Lošo T. The evolutionary origin of orphan genes. Nat Rev Genet. 2011;12:692-702.

6. Tang Y-C, Amon A. Gene copy-number alterations: a cost-benefit analysis. Cell. 2013;152:394-405.

7. Dong S, Raffaele S, Kamoun S. The two-speed genomes of filamentous pathogens: waltz with plants. Curr Opin Genet Dev. 2015;35:57-65.

8. Zolan ME. Chromosome-length polymorphism in fungi. Microbiol Rev. 1995;59:686-98.

9. Sharma R, Mishra B, Runge F, Thines M. Gene loss rather than gene gain is associated with a host jump from monocots to dicots in the smut fungus Melanopsichium pennsylvanicum. Genome Biol Evol. 2014;6(8):2034-49.

10. Ohm RA, Feau N, Henrissat B, Schoch CL, Horwitz BA, Barry KW, et al. Diverse lifestyles and strategies of plant pathogenesis encoded in the genomes of eighteen dothideomycetes fungi. PLoS Pathog. 2012;8(12): e1003037

11. Baroncelli R, Amby DB, Zapparata A, Sarrocco S, Vannacci G, Le Floch $G$, et al. Gene family expansions and contractions are associated with host range in plant pathogens of the genus Colletotrichum. BMC Genomics. 2016;17:555.

12. Gladieux P, Ropars J, Badouin H, Branca A, Aguileta G, de Vienne DM, et al. Fungal evolutionary genomics provides insight into the mechanisms of adaptive divergence in eukaryotes. Mol Ecol. 2014;23:753-73.

13. Faino L, Seidl MF, Datema $E$, van den Berg GC, Janssen A, Wittenberg AH, et al. Single-molecule real-time sequencing combined with optical mapping yields completely finished fungal genome. MBio. 2015;6:e00936-15.

14. Plissonneau C, Stürchler A, Croll D. The evolution of orphan regions in genomes of a fungal pathogen of wheat. MBio. 2016;7:e01231-16.

15. Hurgobin B, Edwards D. SNP discovery using a pangenome: has the single reference approach become obsolete? Biology. 2017:6:21.

16. Tettelin H, Masignani V, Cieslewicz MJ, Donati C, Medini D, Ward NL, et al. Genome analysis of multiple pathogenic isolates of Streptococcus agalactiae: implications for the microbial "pan-genome". Proc Natl Acad Sci U S A. 2005:102:13950-5.

17. Tettelin H, Riley D, Cattuto C, Medini D. Comparative genomics: the bacterial pan-genome. Curr Opin Microbiol. 2008;11:472-7.

18. Croucher NJ, Campo JJ, Le TQ, Liang X, Bentley SD, Hanage WP, et al. Diverse evolutionary patterns of pneumococcal antigens identified by pangenomewide immunological screening. Proc Natl Acad Sci U S A. 2017;114:E357-66.

19. Lefébure T, Stanhope MJ. Evolution of the core and pan-genome of Streptococcus: positive selection, recombination, and genome composition. Genome Biol. 2007:8:R71

20. Lukjancenko O, Wassenaar TM, Ussery DW. Comparison of 61 sequenced Escherichia coli genomes. Microb Ecol. 2010;60:708-20.

21. Chin C-S, Alexander DH, Marks P, Klammer AA, Drake J, Heiner C, et al. Nonhybrid, finished microbial genome assemblies from long-read SMRT sequencing data. Nat Meth. 2013;10:563-9.

22. Laszlo AH, Derrington IM, Ross BC, Brinkerhoff H, Adey A, Nova IC, et al. Decoding long nanopore sequencing reads of natural DNA. Nat Biotech. 2014;32:829-33
23. Golicz AA, Bayer PE, Barker GC, Edger PP, Kim H, Martinez PA, et al. The pangenome of an agronomically important crop plant Brassica oleracea. Nat Commun. 2016;7:13390.

24. Hirsch CN, Foerster JM, Johnson JM, Sekhon RS, Muttoni G, Vaillancourt B, et al. Insights into the maize pan-genome and pan-transcriptome. Plant Cell. 2014;26:121-35.

25. Li Y, Zhou G, Ma J, Jiang W, Jin L, Zhang Z, et al. De novo assembly of soybean wild relatives for pan-genome analysis of diversity and agronomic traits. Nat Biotech. 2014;32:1045-52.

26. Upadhyaya NM, Garnica DP, Karaoglu H, Sperschneider J, Nemri A, Xu B, et al. Comparative genomics of Australian isolates of the wheat stem rust pathogen Puccinia graminis f. sp. tritici reveals extensive polymorphism in candidate effector genes. Puccinia graminis. 2015:5:759.

27. Walkowiak S, Rowland O, Rodrigue N, Subramaniam R. Whole genome sequencing and comparative genomics of closely related Fusarium Head Blight fungi: Fusarium graminearum, F. meridionale and F. asiaticum. BMC Genomics. 2016:17:1014.

28. Valero-Jiménez CA, Faino L, Spring in't Veld D, Smit S, Zwaan BJ, van Kan JA Comparative genomics of Beauveria bassiana: uncovering signatures of virulence against mosquitoes. BMC Genomics. 2016;17:986.

29. Hu X, Xiao G, Zheng P, Shang Y, Su Y, Zhang X, et al. Trajectory and genomic determinants of fungal-pathogen speciation and host adaptation. Proc Natl Acad Sci U S A. 2014;111:16796-801.

30. Yue J-X, Li J, Aigrain L, Hallin J, Persson K, Oliver K, et al. Contrasting evolutionary genome dynamics between domesticated and wild yeasts. Nat Genet. 2017:49:913-24.

31. Jones JDG, Dangl JL. The plant immune system. Nature. 2006:444:323-9.

32. Lo Presti L, Lanver D, Schweizer G, Tanaka S, Liang L, Tollot M, et al. Fungal effectors and plant susceptibility. Annu Rev Plant Biol. 2015;66: 513-45.

33. McDonald BA, Linde C. Pathogen population genetics, evolutionary potential, and durable resistance. Annu Rev Phytopathol. 2002:40:349-79.

34. Raffaele S, Kamoun S. Genome evolution in filamentous plant pathogens: Why bigger can be better. Nat Rev Microbiol. 2012;10:417-30.

35. O'Driscoll A, Kildea S, Doohan F, Spink J, Mullins E. The wheat-Septoria conflict: a new front opening up? Trends Plant Sci. 2014;19:602-10.

36. Cowger C, Hoffer ME, Mundt CC. Specific adaptation by Mycosphaerella graminicola to a resistant wheat cultivar. Plant Pathol. 2000;49:445-51.

37. Torriani SF, Brunner PC, McDonald BA, Sierotzki H. Qol resistance emerged independently at least 4 times in European populations of Mycosphaerella graminicola. Pest Manag Sci. 2009;65:155-62.

38. Zhan J, Pettway RE, McDonald BA. The global genetic structure of the wheat pathogen Mycosphaerella graminicola is characterized by high nuclear diversity, low mitochondrial diversity, regular recombination, and gene flow. Fungal Genet Biol. 2003;38:286-97.

39. Stukenbrock EH, Bataillon T, Dutheil JY, Hansen T, Li R, Zala M, et al. The making of a new pathogen: insights from comparative population genomics of the domesticated wheat pathogen Mycosphaerella graminicola and its wild sister species. Genome Res. 2011;21(12):2157-66.

40. Croll D, Lendenmann MH, Stewart E, McDonald BA. The impact of recombination hotspots on genome evolution of a fungal plant pathogen. Genetics. 2015;201:1213-28.

41. Marshall R, Kombrink A, Motteram J, Loza-Reyes E, Lucas J, HammondKosack KE, et al. Analysis of two in planta expressed LysM effector homologs from the fungus Mycosphaerella graminicola reveals novel functional properties and varying contributions to virulence on wheat. Plant Physiol. 2011;156:756-69.

42. Hartmann FE, Sánchez-Vallet A, McDonald BA, Croll D. A fungal wheat pathogen evolved host specialization by extensive chromosomal rearrangements. ISME J. 2017;11:1189-204.

43. Zhong Z, Marcel TC, Hartmann FE, Ma X, Plissonneau C, Zala M, et al. A small secreted protein in Zymoseptoria tritici is responsible for avirulence on wheat cultivars carrying the Stb6 resistance gene. New Phytol. 2017;214: 619-31.

44. Croll D, Zala M, McDonald BA. Breakage-fusion-bridge cycles and large insertions contribute to the rapid evolution of accessory chromosomes in a fungal pathogen. PLoS Genet. 2013;9:e1003567.

45. Goodwin SB, M'Barek SB, Dhillon B, Wittenberg AHJ, Crane CF, Hane JK, et al. Finished genome of the fungal wheat pathogen Mycosphaerella graminicola reveals dispensome structure, chromosome plasticity, and stealth pathogenesis. PLoS Genet. 2011;7:e1002070. 
46. McDonald BA, Martinez JP. Chromosome length polymorphisms in a Septoria tritici population. Curr Genet. 1991;19:265-71.

47. Croll D, McDonald BA. The accessory genome as a cradle for adaptive evolution in pathogens. PLoS Pathog. 2012;8:e1002608.

48. McDonald MC, McGinness L, Hane JK, Williams AH, Milgate A, Solomon PS. Utilizing gene tree variation to identify candidate effector genes in Zymoseptoria tritici. G3-Genes Genomes Genet. 2016;6:779-91.

49. Lendenmann MH, Croll D, Stewart EL, McDonald BA. Quantitative trait locus mapping of melanization in the plant pathogenic fungus Zymoseptoria tritici. G3. 2014;4:2519-33.

50. Sperschneider J, Gardiner DM, Dodds PN, Tini F, Covarelli L, Singh KB, et al. EffectorP: predicting fungal effector proteins from secretomes using machine learning. New Phytol. 2016;210:743-61.

51. Galagan JE, Selker EU. RIP: the evolutionary cost of genome defense. Trends Genet. 2004;20:417-23.

52. Dean RA, Lichens-Parks A, Kole C. Genomics of Plant-Associated Fungi: Monocot Pathogens. Berlin-Heidelberg: Springer; 2014.

53. Hartmann FE, Croll D. Distinct trajectories of massive recent gene gains and losses in populations of a microbial eukaryotic pathogen. Mol Biol Evol. 2017;34(11):2808-22. https://doi.org/10.1093/molbev/ms×208/3988102.

54. Grandaubert J, Bhattacharyya A, Stukenbrock EH. RNA-seq-based gene annotation and comparative genomics of four fungal grass pathogens in the genus Zymoseptoria identify novel orphan genes and species-specific invasions of transposable elements. G3 (Bethesda). 2015;5:1323-33.

55. Sperschneider J, Dodds PN, Gardiner DM, Manners JM, Singh KB, Taylor JM. Advances and challenges in computational prediction of effectors from plant pathogenic fungi. PLoS Pathog. 2015;11:e1004806.

56. Palma-Guerrero J, Ma X, Torriani SFF, Zala M, Francisco CS, Hartmann FE, et al. Comparative transcriptome analyses in Zymoseptoria tritici reveal significant differences in gene expression among strains during plant infection. Mol Plant Microbe Interact. 2017;30(3):231-44.

57. Cheeseman K, Ropars J, Renault P, Dupont J, Gouzy J, Branca A, et al. Multiple recent horizontal transfers of a large genomic region in cheese making fungi. Nat Commun. 2014;5:2876.

58. Selker EU. Premeiotic instability of repeated sequences in Neurospora crassa. Annu Rev Genet. 1990;24:579-613.

59. Rudd JJ, Kanyuka K, Hassani-Pak K, Derbyshire M, Andongabo A, Devonshire J, et al. Transcriptome and metabolite profiling of the infection cycle of Zymoseptoria tritici on wheat reveals a biphasic interaction with plant immunity involving differential pathogen chromosomal contributions and a variation on the hemibiotrophic lifestyle definition. Plant Physiol. 2015;167:1158-85.

60. Mirzadi Gohari A, Ware SB, Wittenberg AHJ, Mehrabi R, Ben M'Barek S, Verstappen ECP, et al. Effector discovery in the fungal wheat pathogen Zymoseptoria tritici. Mol Plant Pathol. 2015;16:931-45.

61. Win J, Chaparro-Garcia A, Belhaj K, Saunders DG, Yoshida K, Dong S, Schornack S, Zipfel C, Robatzek S, Hogenhout SA, Kamoun S. Effector biology of plant-associated organisms: concepts and perspectives. Cold Spring Harb Symp Quant Biol. 2012;77:235-47.

62. Albalat R, Cañestro C. Evolution by gene loss. Nat Rev Genet. 2016;17:379-91.

63. Brown JKM, Chartrain L, Lasserre-Zuber P, Saintenac C. Genetics of resistance to Zymoseptoria tritici and applications to wheat breeding. Fungal Genet Biol. 2015;79:33-41.

64. Mclnerney JO, McNally A, O'Connell MJ. Why prokaryotes have pangenomes. Nat Microbiol. 2017;2:17040.

65. Carvunis A-R, Rolland T, Wapinski I, Calderwood MA, Yildirim MA, Simonis N, et al. Proto-genes and de novo gene birth. Nature. 2012;487:370-4.

66. Yao W, Li G, Zhao H, Wang G, Lian X, Xie W. Exploring the rice dispensable genome using a metagenome-like assembly strategy. Genome Biol. 2015;16:187.

67. Donati C, Hiller NL, Tettelin H, Muzzi A, Croucher NJ, Angiuoli SV, et al. Structure and dynamics of the pan-genome of Streptococcus pneumoniae and closely related species. Genome Biol. 2010;11:R107.

68. Zhan J, Linde CC, Jürgens T, Merz U, Steinebrunner F, McDonald BA. Variation for neutral markers is correlated with variation for quantitative traits in the plant pathogenic fungus Mycosphaerella graminicola. Mol Ecol. 2005; 14:2683-93.

69. Walker BJ, Abeel T, Shea T, Priest M, Abouelliel A, Sakthikumar S, Cuomo CA Zeng Q, Wortman J, Young SK, Earl AM. Pilon: an integrated tool for comprehensive microbial variant detection and genome assembly improvement. PLoS One. 2014;9:e112963.
70. Hoff KJ, Lange S, Lomsadze A, Borodovsky M, Stanke M. BRAKER1: Unsupervised RNA-Seq-Based genome annotation with GeneMark-ET and AUGUSTUS. Bioinformatics. 2016:32:767-9.

71. Bolger AM, Lohse M, Usadel B. Trimmomatic: a flexible trimmer for Illumina sequence data. Bioinformatics. 2014;30:2114-20.

72. Trapnell C, Pachter L, Salzberg SL. TopHat: discovering splice junctions with RNA-Seq. Bioinformatics. 2009;25:1105-11.

73. Stanke M, Schöffmann O, Morgenstern B, Waack S. Gene prediction in eukaryotes with a generalized hidden Markov model that uses hints from external sources. BMC Bioinformatics. 2006;7:62.

74. Slater GSC, Birney E. Automated generation of heuristics for biological sequence comparison. BMC Bioinformatics. 2005;6:31.

75. Anders S, Pyl PT, Huber W. HTSeq-a Python framework to work with high-throughput sequencing data. Bioinformatics. 2015;31:166-9.

76. Robinson MD, McCarthy DJ, Smyth GK. edgeR: a Bioconductor package for differential expression analysis of digital gene expression data. Bioinformatics. 2010;26:139-40.

77. Simão FA, Waterhouse RM, loannidis P, Kriventseva EV, Zdobnov EM. BUSCO: assessing genome assembly and annotation completeness with single-copy orthologs. Bioinformatics. 2015;3119:3210-2.

78. Jones P, Binns D, Chang H-Y, Fraser M, Li W, McAnulla C, et al. InterProScan 5: genome-scale protein function classification. Bioinformatics. 2014;30:1236-40.

79. Petersen TN, Brunak S, von Heijne G, Nielsen H. SignalP 4.0: discriminating signal peptides from transmembrane regions. Nat Meth. 2011;8:785-6.

80. Käll L, Krogh A, Sonnhammer ELL. A combined transmembrane topology and signal peptide prediction method. J Mol Biol. 2004;338:1027-36.

81. Krogh A, Larsson B, von Heijne G, Sonnhammer E. Predicting transmembrane protein topology with a hidden Markov model: application to complete genomes. J Mol Biol. 2001;305:567-80.

82. Altschul SF, Gish W, Miller W, Myers EW, Lipman DJ. Basic local alignment search tool. J Mol Biol. 1990;215:403-10.

83. Miele $V$, Penel $S$, Duret L. Ultra-fast sequence clustering from similarity networks with SiLiX. BMC Bioinformatics. 2011;12:116.

84. Guy L, Kultima JR, Andersson SGE. genoPlotR: comparative gene and genome visualization in R. Bioinformatics. 2010;26:2334-5.

85. Schotanus K, Soyer JL, Connolly LR, Grandaubert J, Happel P, Smith KM, et al. Histone modifications rather than the novel regional centromeres of Zymoseptoria tritici distinguish core and accessory chromosomes. Epigenetics Chromatin. 2015;8:41.

86. Song Q, Smith AD. Identifying dispersed epigenomic domains from ChIP-Seq data. Bioinformatics. 2011;27:870-1.

87. Linde CC, Zhan J, McDonald BA. Population structure of Mycosphaerella graminicola: from lesions to continents. Phytopathology. 2002;92:946-55.

88. Torriani SFF, Stukenbrock EH, Brunner PC, McDonald BA, Croll D. Evidence for extensive recent intron transposition in closely related fungi. Curr Biol. 2011;21:2017-22.

89. Langmead B, Salzberg SL. Fast gapped-read alignment with Bowtie 2. Nat Methods. 2012;9:357-9.

90. McKenna A, Hanna M, Banks E, Sivachenko A, Cibulskis K, Kernytsky A et al. The Genome Analysis Toolkit: a MapReduce framework for analyzing next-generation DNA sequencing data. Genome Res. 2010;20:1297-303.

91. Cingolani P, Platts A, Wang LL, Coon M, Nguyen T, Wang L, et al. A program for annotating and predicting the effects of single nucleotide polymorphisms, SnpEff. Fly (Austin). 2012;6:80-92.

92. Cingolani P, Patel VM, Coon M, Nguyen T, Land SJ, Ruden DM, et al. Using Drosophila melanogaster as a model for genotoxic chemical mutational studies with a new program. SnpSift Front Genet. 2012;3:35.

93. Danecek P, Auton A, Abecasis G, Albers CA, Banks E, DePristo MA, et al. The variant call format and VCFtools. Bioinformatics. 2011;27:2156-8.

94. Abyzov A, Urban AE, Snyder M, Gerstein M. CNVnator: an approach to discover, genotype, and characterize typical and atypical CNVs from family and population genome sequencing. Genome Res. 2011;21:974-84.

95. Quinlan AR, Hall IM. BEDTools: a flexible suite of utilities for comparing genomic features. Bioinformatics. 2010;26:841-2.

96. Falcon S, Gentleman R. Using GOstats to test gene lists for GO term association. Bioinformatics. 2007;23:257-8. 